\title{
Changes in Availability and Affordability of Therapeutic Monoclonal Antibodies After New Medical Reform in Hubei Province, China
}

\section{Yufeng Ding}

Tongji Hospital of Tongji Medical College of Huazhong University of Science and Technology Jinwen Zhang

Tongji Hospital of Tongji Medical College of Huazhong University of Science and Technology Meijun Xia

Huazhong University of Science and Technology Tongji Medical College

\section{Guangjie Wu}

Tongji Hospital of Tongji Medical College of Huazhong University of Science and Technology

\section{Weijie Li}

Tongji Hospital of Tongji Medical College of Huazhong University of Science and Technology

\section{Da Feng}

Huazhong University of Science and Technology Tongji Medical College

Shiwei Gong ( $\nabla$ shwgong@hust.edu.cn )

Huazhong University of Science and Technology https://orcid.org/0000-0003-3556-8512

\section{Research}

Keywords: Availability, Affordability, Monoclonal antibody, New medical reform, China

Posted Date: April 25th, 2020

DOI: https://doi.org/10.21203/rs.3.rs-23749/v1

License: (9) This work is licensed under a Creative Commons Attribution 4.0 International License. Read Full License 


\section{Title page}

Title: Changes in Availability and Affordability of Therapeutic Monoclonal Antibodies After New Medical Reform in Hubei Province, China

\section{Author details}

Yufeng Ding ${ }^{1}$, Jinwen Zhang ${ }^{1}$, Meijun Xia ${ }^{2}$, Guangjie $\mathrm{Wu}^{1}$, Weijie $\mathrm{Li}^{1}$, Da Feng ${ }^{2}$, Shiwei Gong*2

1 Department of pharmacy, Tongji Hospital, Tongji Medical College of Huazhong University of Science and Technology, Wuhan 430030, Hubei, China

${ }^{2}$ Department of Pharmacy Business and Administration, School of Pharmacy, Tongji Medical College of Huazhong University of Science and Technology, Wuhan 430030, Hubei, China

\section{*Corresponding author: Shiwei Gong}

Department of Pharmacy Business and Administration, School of Pharmacy, Tongji

Medical College of Huazhong University of Science and Technology, Wuhan 430030, Hubei, China

\section{Email: shwgong@hust.edu.cn}

\section{Ethics approval and consent to participate}

The hospital survey was approved by the Medical Ethics Committee of Tongji

Medical College of Huazhong University of Science \& Technology (No: S005)

\section{Consent for publication}

Not applicable.

\section{Availability of data and material}

Requests for access to data should be addressed to Shiwei Gong. (shwgong@hust.edu.cn).

\section{Competing interests}

The authors declare that they have no competing interests.

\section{Funding}

This work was supported by the grants from the National Natural Science Foundation of China (No: 70903025, No: 71373089).

\section{Authors' contributions:}

Yufeng Ding, Shiwei Gong designed this study and organized two surveys. Yufeng Ding, Guangjie Wu, Da Feng, Weijie Li, Meijun Xia, Jinwen Zhang collected all survey data. Guangjie Wu, Jinwen Zhang revised critically the manuscript for important intellectual content. Shiwei Gong, Meijun Xia analyzed and interpreted the survey data. Shiwei Gong, Meijun Xia contributed to writing the drafting of the manuscript. All authors read and approved the final manuscript.

\section{Acknowledgements:}

The authors would like to thank to all pharmacists and pharmacy experts and managers of 50 hospitals in Hubei province that helped us fill out a series of forms in the survey. 


\section{$1 \quad$ Main document}

\section{Abstract}

3 Background: In 2017, China launched a new round of medical reform (NMR) to solve

4 the problems of "expensive and difficult to use drugs" for patients. The expensive and

5 high-value therapeutic monoclonal antibodies (mAbs) have become the focus of attention. Promoting the availability and affordability of mAbs is an important way to improve the quality of life and health equity of patients.

8 Methods: We used a standard method developed by the World Health Organization to

9 conduct two surveys on mAbs' availability and price before and after the NMR in the

10 public general hospitals in Hubei province. By interviewing experts from these hospital 11 pharmacies, we identified the factors that contributed to low accessibility and determined the effectiveness of NMR.

Results: After the NMR, we found the average availability of 13 mAbs increased by $6.7 \%$ in the survey hospitals of Hubei Province. The median per unit price of $10 \mathrm{mAbs}$ dropped by $34.3 \%$. The average cost of a treatment cycle of $10 \mathrm{mAbs}$ dropped from an equivalent of 529 days to 151 days of daily disposable income for a middle-income resident. Only trastuzumab and bevacizumab showed a significant decrease in price and a significant increase in availability, simultaneously. High price, lack of clinical demand and hospital drug procurement list, low income of patients, limited coverage and reimbursement of National Basic Medical Insurance (NBMI) for mAbs, and lack of incentives for generic mAbs were the main obstacles to the accessibility of mAbs. The drug negotiation mechanism of NBMI and the promotion of consistency evaluation of generic and original drugs can effectively promote the accessibility of mAbs.

Conclusions: The availability and affordability of mAbs in Hubei Province have improved significantly, but still at a low level. There is currently no synergy between 
1 the policies of the NMR to promote the accessibility of the mAbs. In the future, we

2 should implement $\mathrm{R} \& \mathrm{D}$ incentives for high-quality mAbs and generics, conduct

3 medication evaluation and education of therapeutic mAbs, and design a special medical

4 insurance payment method.

5 Keywords: Availability; Affordability; Monoclonal antibody; New medical reform;

6 China

$7 \quad$ Introduction

8 Monoclonal antibody (mAb) refers to an immunoglobulin that binds to a specific

9 epitope on an antigen to exert its immunological effect [1] . In 1986, the first therapeutic

10 mAb Orthoclone OKT3 was approved by the US FDA for the prevention of renal

11 transplant rejection. Since then, the development of therapeutic mAbs has flourished

12 into powerful human biological products for providing new and effective therapies for

13 cancer, autoimmune, cardiovascular and infectious diseases, etc [2]. Human mAbs also

14 began to be used as candidate therapeutics against viruses, such as SARS-CoV, MERS-

$15 \mathrm{CoV}$, and Ebola virus [3]. According to the China's Diagnosis and Treatment Guideline

16 for COVID-19 pneumonia (seventh edition), tocilizumab is used to suppress cytokine

17 storms [4]. With the research advances, highly specific and safe human MAbs have

18 been applied in the treatment [5]. MAb drugs have shown very important therapeutic

19 value in the treatment of human life-threatening diseases.

20 The approval rate and sales of mAbs have increased dramatically in the market [6].

21 From 1986 to May 2017, the United States and Europe approved a total of 76

22 therapeutic mAbs for commercial sales [7]. In 2017, the global sales revenue of all mAb

23 drugs totaled 123.3 billion USD, accounting for $65.6 \%$ of total global

24 biopharmaceutical sales. MAbs have become a single product category with the highest

25 market potential.[8]Between 2014 and 2017, adalimumab, infliximab, rituximab, 
trastuzumab, and bevacizumab achieved sales of $\$ 62.6$ billion, $\$ 35.6$ billion, $\$ 29.1$ billion, \$27.1 billion, and \$27 billion USD, respectively, and the five drugs are all among the top 10 best-selling drugs in the world [8]. In 2002, Wuhan Institute of Biological Products Co., Ltd. launched China's first mAb drug, mouse mAb against human CD3 antigen of T lymphocyte. By 2014, the market size of therapeutic mAbs in China reached $¥ 5.034$ billion CNY (USD 0.823 billion).

However, patients in China still do not access to all the mAb drugs available elsewhere and the most appropriate medication. Rong Jiang's research showed that only 11 of 83 innovative mAb drugs marketed in other countries were also launched in China, indicating a huge drug lag compared to other developed countries in 2017 [9]. Xu et al. performed an overall analysis of drug use from 2014 to 2016 in sample hospitals in 22 cities (regions) in China and also found no mAb ranked among the top ten drugs in terms of sales [10]. In addition, although most therapeutic mAbs have significant clinical value, most are also expensive. For example, the price of adalimumab (Humira, $40 \mathrm{mg} / 0.4 \mathrm{ml}$ ) is approximately USD 5490 per pen injection kit for two ea [11]. China is also facing with the problem of high drug prices. Financial unaffordability is equivalent to no drug available to the patient.

In order to improve the availability and affordability of high-value drugs for patients in China, the State Council issued the "Thirteenth Five-Year Plan for deepening the medical reforms" on January 9, 2017. A series of reform measures closely related to the accessibility of therapeutic mAbs were proposed, including zero-markup on drug prices in public hospitals, limits on the proportions of drug expenditure to total medical expenditure in public hospitals, and a "two-invoice restriction system" from the pharmaceutical factory to public hospital, acceleration of the review and approval of new drugs against malignant tumors and rare diseases, the consistency evaluation 
1 policy of generic drug and corresponding original drug, and the price negotiation and centralized procurement policies for drugs covered by NBMI [12].

In 2016, China's pharmaceutical retail market sales totaled $¥ 1.081$ trillion CNY, among which the pharmaceutical procurement by medical institutions totaled $¥ 767.3$ billion CNY, accounting for $71.0 \%$ of the pharmaceutical retail sales, suggesting that medical institutions are the main channel for drug supply in China [13]. To understand the impact of the NMR on the availability and affordability of therapeutic mAbs marketed in China, we took 2017 as the dividing point and surveyed changes in the availability and affordability of these mAbs in Hubei public hospitals between May 2016 and November 2018. We also explored the influencing factors that contributed to low accessibility and evaluated the value of NMR policies for increasing the availability and affordability of the mAbs.

\section{Methods}

\section{Sampling}

Hubei province is located in central China with 17 prefecture-level cities. In 2018, Hubei province had a population of 59.17 million, a GDP per capita of $¥ 66,531 \mathrm{CNY}$ (compared to the national average of $¥ 64,644 \mathrm{CNY}$ ), and an annual per capita disposable income per household of $¥ 25,815 \mathrm{CNY}$ (compared to a national average of $¥ 28,166 \mathrm{CNY}$ ), making it a province with economic development slightly higher than average [14]. Taken into account the differences in geographical location and economic levels of cities in Hubei province, the surveyed hospitals were selected from prefecturelevel cities of three distinct economic levels from five locations: East, south, west, north, and central. In each city, secondary and tertiary public general hospitals were investigated. China's primary hospitals mainly provide essential drugs and do not cover therapeutic mAbs, and therefore they were not selected. For the selection of secondary 
1 and tertiary hospitals in each city, all hospitals were surveyed in cities with fewer than

2 four public general hospitals. In cities with more than four public general hospitals, all

3 hospitals were enumerated, and four secondary and tertiary general hospitals were

4 randomly selected.

5 In May 2016, we selected five prefecture-level cities at three economic levels:

6 Wuhan, Yichang, Huanggang, Xiangyang, and Jingzhou. We identified 15 secondary

7 hospitals and 17 tertiary hospitals, for a total of 32 hospitals. In November 2018, to

8 ensure that the collected data can fully represent the status of the hospitals in Hubei

9 province, we selected eight prefecture-level cities, namely Wuhan, Yichang,

10 Huanggang, Xiangyang, Jingzhou, Xianning, Suizhou, and Enshi, and identified 24

11 secondary hospitals and 30 tertiary hospitals, for a total of 54 hospitals. Information on

12 the economic and demographic characteristics of eight cities was summarized in the

13 additional file1.

14 Investigated drugs

15 As of March 2016, $13 \mathrm{mAb}$ drugs (excluding fusion protein antibody drugs) were 16 approved by the NMPA of China for marketing [15]. Table 1 lists the general 17 information on the indications, targets, and supply sources of the $13 \mathrm{mAbs}$ [16]. Each $18 \mathrm{mAb}$ drug was investigated for the availability and prices of the original brand-name 19 drug and generic drug in the hospital. Among the available generic drugs, we selected 20 the generic drug with the lowest price in the hospitals surveyed.

\section{Data collection and statistical analysis}

22 A semi-structured questionnaire on therapeutic mAbs was designed according to the 23 WHO / HAI Drug Standard Survey Method, including information on the availability 24 and price of the original drug and the lowest-priced generic drug [17]. The questionnaire 25 was distributed to the person in charge at the hospital pharmacy by mail or by email. 
1 The questionnaire was completed by a pharmacist with a senior technical title. The

2 survey data were collected from May to June 2016 and from November to December 32018 .

The evaluation criteria for drug availability and affordability were designed according to the WHO/HAI Drug Standard Survey Method. The standard for evaluating drug availability is the ratio of the number of medical institutions equipped with the drug to the total number of medical institutions surveyed, i.e., drug availability $=$ Number of medical institutions equipped with the drug/Total number of medical institutions surveyed $\times 100 \%$. The following criteria from the WHO were used to describe the availability of therapeutic mAbs [18]:

- Absent ( $0 \%$ of hospitals): not found in any hospital surveyed;

- Very low $(<30 \%$ of hospitals): very difficult to find;

- Low (30-49\% of hospitals): somewhat difficult to find;

- Fair high (50-80\% of hospitals): available in many hospitals;

- Very high ( $>80 \%$ of hospitals): good availability.

The standard for assessing the affordability of medicines is the standard cost of drugs for a course of treatment and its equivalence to multiples of the minimum daily wage of non-technical government workers, that is, the affordability of medicines is equivalent to the cost of medicines for a course equivalent to the cumulative number of days at minimum daily wages for non-technical government workers. Since there is no statistical data on the minimum daily wage of non-technical government workers in Hubei Province, our study uses the average daily disposable income of urban and rural residents in Hubei Province instead of the minimum daily wage of non-technical workers [19]. Therefore, if the cost of one course of treatment using therapeutic mAbs does not exceed the daily disposable income of the patient, the drug is considered to be 
affordable (see Formula 1).

Affordability of drug $=($ Defined daily dose of the drug $\times$ retail unit price of the drug $\times$ days per treatment course) $\div$ average disposable income. (Formula 1)

Defined daily dose (DDD) refers to the standard dose measurement unit of an average daily maintenance dose for adults with major indications. The DDD values of some mAbs in the study were reported by the WHO [20]. The DDD values of some other mAbs were not found in the WHO DDD index. According to the recommended dosage of the corresponding drug package, we calculated the maintenance dose for a $70 \mathrm{~kg}$ adult and determined the course of treatment according to the instructions of the drugs [21]. The course of treatment was defined based on WHO regulations: seven days for acute symptoms, and 30 days for chronic symptoms [17]. The retail unit price of a drug is the median retail price per unit of a mAb sold by the hospitals surveyed. In this study, the median affordability value of a drug can be calculated by the retail price of the drug collected from two hospitals or more. In 2016, the per capita daily disposable income of Hubei residents was $¥ 59.7 \mathrm{CNY}$, compared with $¥ 70.7 \mathrm{CNY}$ in 2018 [14].

We then used the Chi-Square test to calculate the significance of the difference in the availability and price distribution of $13 \mathrm{mAbs}$ before and after the NMR, respectively.

\section{Results}

\section{General information on selected hospitals}

A total of 28 general hospitals surveyed in 2016 sent back the completed questionnaire (the response rate was $87.5 \%$ ), which comprised 11 secondary hospitals and 17 tertiary hospitals. Of the 54 general hospitals surveyed in 2018, a total of 50 sent back the completed questionnaire (response rate was 92.5\%), including 20 secondary hospitals and 30 tertiary hospitals. 
1 Changes in the availability of therapeutic mAbs after the NMR

2 Table 2 presents the changes in the availability of $13 \mathrm{mAbs}$ in the general hospitals of

3 Hubei province before and after the NMR. Of $13 \mathrm{mAbs}, 11$ were available and two

4 were unavailable before and after NMR. After the NMR, the average availability of 13

5 mAbs was increased by $6.7 \%$, from $10.4 \%$ in 2016 to $17.1 \%$ in 2018 and was still at

6 the very low level. The availability of rituximab, trastuzumab, bevacizumab was

7 increased by greater than $10 \%$.

In 2016 , the availability of $13 \mathrm{mAbs}$ was less than $30 \%$. In 2018 , there were three rituximab. The availability of trastuzumab and bevacizumab showed significant increase by $24.6 \%\left(\chi^{2}=4.6, \mathrm{df}=1, P=0.031\right)$ and $29.3 \%\left(\chi^{2}=7.4, \mathrm{df}=1, \mathrm{P}=0.007\right)$, respectively. However, four Chinese domestic mAbs before and after the NMR showed unavailability or very low availability (1.8\% in 2016 versus 3\% in 2018). (see 14 additional file2)

\section{Changes in the price level of therapeutic mAbs after the NMR}

The median retail price of $10 \mathrm{mAbs}$ available in 2016 in the 28 hospitals was $¥ 7199.5$ $\mathrm{CNY}$, and the median unit price was $¥ 581.8 \mathrm{CNY} / \mathrm{mg}$ (Table 3). Among them, the drug with the highest unit price was ranbizumab, reaching a price as high as $¥ 4877.8 \mathrm{CNY}$ / mg, followed by basiliximab and adalimumab. In 2018, the median retail price of 10 mAbs in 50 hospitals was $¥ 4303.0 \mathrm{CNY}$, and the median unit price was $¥ 362.8 \mathrm{CNY}$ / mg, with a decrease of $¥ 2896.5 \mathrm{CNY}$ and $¥ 219 \mathrm{CNY} / \mathrm{mg}$, respectively. The prices of the ten drugs were dropped by $34.3 \%$ overall.

After the NMR, unit prices of six drugs have all fallen by more than $40 \%$, including cetuximab (70.0\%), trastuzumab (66.9\%), bevacizumab (63.4\%), nimotuzumab 25 (51.3\%), ranibizumab (41.6\%), and rituximab (40.1\%). But the prices for the other four 
mAbs, namely adalimumab, infliximab, tocilizumab, and basiliximab, have only fallen by less than 5\%. Chi-Square tests showed the unit price distribution of cetuximab $\left(\chi^{2}=\right.$ 11.6, $\mathrm{df}=5, p=0.041)$, rituximab $\left(\chi^{2}=27.3, \mathrm{df}=9, p=0.001\right)$, trastuzumab $\left(\chi^{2}=25.3\right.$, $\mathrm{df}=7, p=0.001)$, bevacizumab $\left(\chi^{2}=18.7, \mathrm{df}=5, p=0.002\right)$ and ranibizumab $\left(\chi^{2}=\right.$ 18.0, $\mathrm{df}=5, p=0.003$ ) has dropped significantly before and after the NMR. (see additional file3)

\section{Changes in the affordability of therapeutic mAbs after the NMR}

We calculated the affordability of $10 \mathrm{mAbs}$ available in Hubei general hospitals in 2016 and in 2018 (Table 4). The average cost per course of treatment for the $10 \mathrm{mAb}$ drugs available in general hospitals in 2016 was $¥ 40,583.6 \mathrm{CNY}$. The cost of one treatment cycle is equivalent to 680 days of daily disposable income for a middle-income resident in Hubei province. After the NMR, the average cost each course and the number of burden days of these mAbs were decreased by $¥ 18,550.5 \mathrm{CNY}$ and 382 days, respectively, to $¥ 21093.0 \mathrm{CNY}$ and 298 days. This demonstrated a significant decrease in drug spending. In particular, the number of burden days of rituximab and cetuximab was reduced by 1135 days and 1028 days, respectively. However, the treatment costs of the mAbs were still very high. For example, the treatment cost of rituximab could be as high as $¥ 81,798.8 \mathrm{CNY}$, which is 1157 days of daily disposable income for a middleincome resident in Hubei province.

After the NMR, in addition to basiliximab, five new mAbs were included in the Class B drugs list of basic medical insurance in Hubei province through the national drug negotiation mechanism, covering rituximab, trastuzumab, bevacizumab, nimotuzumab, and ranibizumab. At present, the actual compensation ratio for the Class B drug in China is generally around 70\% [22]. Therefore, the out-of-pocket ratio of the six mAbs covered by our study that fall under the Class B list was set at $30 \%$. Our 
1 results showed that after the costs of the six mAbs were reimbursed by medical

2 insurance, the average actual out-of-pocket cost was $¥ 7,467 \mathrm{CNY}$, which was reduced

3 by $¥ 17,424.3 \mathrm{CNY}$ compared with the pre-reimbursement cost. The average number of

4 burden days of the six mAbs after medical insurance reimbursement was 106 days,

5 which was 246 days less than that before the NMR. (see additional file4)

6 After insurance reimbursement, the average burden days for one course of

7 treatment with 10 therapeutic mAbs was 151 days, which was 529 days less than that

8 before the NMR. Among these, the number of burden days for one course of treatment

9 was reduced by total 1945 days for rituximab and 1123 days for bevacizumab.

10 Barriers to the accessibility of mAb drugs after NMR

11 As shown in Tables 2 and 4, although the availability and affordability of mAbs were improved following the NMR, they were still at low level. Figure 1 shows the recognition rates of pharmacy experts from the 50 surveyed hospitals on different barriers that affect the availability and affordability of $13 \mathrm{mAbs}$. The top five barriers to the availability of $13 \mathrm{mAbs}$ were high price, low clinical demand, no drug

16 procurement list in hospitals, low patient income, restrictions of drug use in the clinical departments and lack of suppliers. Together, these five factors accounted for $57.2 \%$ of the total responses. availability. Compared with imported mAbs, the following three obstacles are greater

21 for the domestic drugs, including low clinical demand, no drug procurement list in hospitals, not included in the drug centralized bidding platform of public hospitals. However, imported mAbs show more obstacles in logistics and distribution than domestic mAbs. (see additional file5) 
The surveyed pharmacy experts believed that the following top five reasons contributed to the unaffordability of mAbs among the general patient population.

They were low income of patients and their families, limited coverage and reimbursement of NBMI for therapeutic mAbs, lack of national incentive policies for generic mAbs, lack of channels for hospitals to apply for drug donations, lack of enthusiasm of pharmaceutical companies for research and development (R \& D) and production of therapeutic mAbs, and lack of national price reduction mechanism for mAbs. These five factors collectively accounted for $83.9 \%$ of the total responses.

\section{Effectiveness of the NMR in promoting the accessibility of therapeutic mAbs}

Figure 2 shows the opinions of 50 surveyed pharmacy experts on the effectiveness of six NMR policies to promote the accessibility of therapeutic mAbs. The experts rated "four" for the impacts of drug price negotiation mechanism of the NBMI and the consistency evaluation of generic and original drug on the availability and affordability of mAbs, suggesting that these policies were "useful". The policy of accelerating the review and approval of new drugs was also useful for promoting the availability of $\mathrm{mAb}$ drugs (median value of the impact $=4$ ). The zero-markup policy for public hospital drugs showed some effect on promoting the affordability of mAb drugs. Other new medical reform policies showed a somewhat useful impact on promoting $\mathrm{mAb}$ availability. In general, the median impact index of the six NMR policies on the availability and affordability of therapeutic mAbs were 3.5 and 3.0, respectively. More than $60 \%$ of experts believed that promoting drug price negotiations and evaluating the consistency of generic and original drugs could effectively improve the accessibility of therapeutic mAbs. (see additional file6)

Experts particularly pointed out that the current implementation of the zero-markup policy for drug pricing and the drug cost proportional control policy in public hospitals 
1 have made mAbs with high prices and low settlement rates of medical insurance fund

2 show no benefits but increased the risk of management costs and control fees. Therefore,

3 even if the mAbs are included in the medical insurance catalog, they are still not

4 guaranteed for clinical use in hospitals.

\section{Discussion}

6 China launched the "Thirteenth Five-Year Plan" for new medical and health system reforms in 2017. For the first time, our research reported the real-world impact of these reform policies on the availability and affordability of high-priced therapeutic mAbs in the general public hospitals. Our results have suggested the following findings:

After the NMR, the availability of therapeutic mAbs in China in Hubei general hospitals was improved, but the overall availability was still limited. The $13 \mathrm{mAbs}$ available on the Chinese market in 2016 had an average increase of over $6 \%$ in availability in 2018. Of these drugs, the availability of trastuzumab and bevacizumab was significantly increased. Among them, the availability of domestic mAbs was particularly low.

After the NMR, the average per unit median price of the $10 \mathrm{mAbs}$ available in 2016 was decreased by $34.3 \%$. Of these, the unit price of the three mAbs have shown a substantial and significant decline, falling by $70.0 \%$ for cetuximab, $66.9 \%$ for trastuzumab, and $63.4 \%$ for bevacizumab, respectively. However, the unit prices of other four mAbs were only decreased by less than $5 \%$.

After the NMR, the cost of a single treatment course for the 10 mAbs listed in 2016 was equivalent to 151 days (6 to 350 days) of the daily disposable income of middleincome patients, and the average burden days was reduced by 529 days ( 17 - 1945 days) following the NMR. The ten mAbs were largely still not affordable in 2018.

Hospital pharmacy experts believed that the main obstacles affecting the 
1 accessibility of mAb s in Hubei general hospitals were high prices, low clinical demand,

2 and no drug procurement catalog in hospitals. The main obstacles affecting the

3 affordability of mAbs were low household income of patients, limited coverage and

4 reimbursement of the NBMI for these mAbs, and the lack of a national incentive policy

5 for generic mAbs. With NMR, the drug negotiation mechanism of NBMI and the

6 promotion of consistency evaluation of generic and original drugs can increase the

7 availability and affordability of therapeutic mAbs effectively. Unexpectedly, the

8 combination of the zero-markup policy for drug pricing and the drug cost proportional

9 control policy has shown a negative effect on the availability of mAbs in public

10 hospitals.

11 Furthermore, how do the mAbs differ from other types of drugs in terms of availability, affordability, and barriers? In 2013, Xi et al. performed a survey on 50 original brand-name essential drugs and generic essential drugs in the secondary and tertiary hospitals of Jiangsu Province and found that the availability rates were $36.8 \%$ and $32.6 \%$, respectively. The average monthly treatment cost of 50 essential drugs was equivalent to 1.3 days of wages for non-technical government workers. Most generic drugs used for standard treatments were affordable, and this affordability was attributed to the improvement in living standards and comprehensive medical insurance coverage of Jiangsu residents [23]. Obviously, compared with mAbs, the availability of essential drugs was about twice as high, and affordability was 100 times higher. For low-priced essential drugs, pharmaceutical companies, hospitals, and doctors are not willing to promote and use them [23]. However, the essential drug policy enforced by the Chinese government guarantees the availability and affordability of essential drugs, with approaches including high coverage and reimbursement requirements of medical insurance, restrictions on the maximum retail price, and the release of essential drug list 
1 and clinical guidelines. In contrast, mAbs are expensive, with low levels of coverage and reimbursement in medical insurance and primitive guidelines of clinical use. These factors have greatly limited the hospital use and accessibility of mAb drugs.

In 2016, Liu et al. found that fast-acting insulin, long-acting insulin, and premixed insulin were highly accessible at rates of $70 \%, 80 \%$, and $90 \%$, respectively, among the 30 public hospitals in Wuhan and five other cities in Hubei Province. The average cost of a month of treatment for patients receiving insulin equated to 4-16 days of wages, indicating a low affordability. The authors believed that the high price of insulin may impose a heavy financial burden on patients. Also, the reimbursement of NBMI was very limited, and the copay rate for their own medicines was still high. Studies have shown that less than $14 \%$ of the direct costs of drugs dispensed through outpatient outlets were covered patients' medical insurance [24]. Compared with mAbs, the availability of insulin for treating common diseases of diabetes was about 4 times higher and the affordability was about 10 times higher. Rather, people have mainly expressed concerns about the unaffordability of insulin. Although in the past 10 years, more than half of the top ten drugs sold worldwide were mAbs, the clinical application of mAbs in China is still limited [8,25]. In this study, our results showed that there were other factors besides the obstacles mentioned above, such as the lack of evidence-based medical investigations of mAbs, the lack of guidelines for the clinical use of mAbs, the lack of training of the standardized use of mAb for doctors, limited awareness of doctors, and lack of scientific and technological exchanges and technical support for mAbs. These factors represent important differences in accessibility between commonly used drugs and mAbs.

\section{Conclusions}

After the NMR, the availability and affordability of therapeutic mAbs in Hubei 
1 province have improved significantly, but the levels are still low. There seems to be no

2 synergy between these reform policies to promote the availability and affordability of

3 the mAbs in the public hospitals. Therefore, in order to optimize the NMR policy

4 system and further increase the accessibility of mAbs, it is necessary to take the

5 following measures, including substantially promoting the technological advancement

6 of research and production for high-quality mAbs and generics, strengthening evidence-

7 based pharmacoeconomical evaluation of mAbs in the Chinese population, establishing

8 clinical treatment guidelines of mAbs, and increasing the training of doctors on mAbs

9 use and the level of medical insurance payment for mAbs.

$10 \quad$ Limitations

11 To exactly fulfill the WHO's survey requirements on the availability and affordability

12 of medicines, the survey should further include regional public hospitals and private retail pharmacies. In 2016, 71\% of China's drug sales came from medical institutions. Due to the high price of therapeutic mAbs and no settlement of medical insurance fund in retail pharmacies, it is highly risky for private retail pharmacies to maintain the supply of therapeutic mAbs, and relatively few retail pharmacies provide the drugs based on the results of our preliminary survey in Wuhan city in 2016. Therefore, our study only conducted data surveys in public hospitals and did not investigate private retail pharmacies. As a result, the mAb availability can be biased in Hubei Province. minimum daily wage of non-technical government workers to calculate drug affordability. Since the disposable income per capita of average residents is generally lower than the minimum daily wage of non-technical government workers, the affordability of therapeutic mAbs could be under-estimated. 
1 on the day of the survey at each hospital, and thus cannot reflect the average monthly or yearly availability of mAbs and their prices.

3 Finally, the position of the people surveyed may bias the analysis of the reasons for

4 the unavailability and unaffordability of $\mathrm{mAbs}$ and the determination of the contribution

5 of the NMR. The experts surveyed were selected only from the pharmacies of general

6 hospitals in the Hubei province, excluding pharmaceutical companies, medical

7 insurance and government agencies.

\section{$9 \quad$ List of abbreviations}

10 Defined daily dose (DDD)

11 Lowest-price generics (LPGs)

12 Monoclonal antibodies(mAbs)

13 Mann-Whitney U test (MW UTest)

14 New medical reform (NMR)

15 National Basic Medical Insurance (NBMI)

16 Health Action International (HAI)

17 Originator brands $(\mathrm{OBs})$

18 Research and development (R \& D)

19 World Health Organization (WHO)

\section{References:}

22 1. Posner J, Barrington P, Brier T, Datta-Mannan A. Monoclonal antibodies: past, present and 23 future. Handb Exp Pharmacol. 2019;260:81-141.

24 2. Leavy O. Therapeutic antibodies: past, present and future. NAT REV IMMUNOL. $25 \quad 2010 ; 10: 297$.

26 3. Jin Y, Lei C, Hu D, Dimitrov DS, Ying T. Human monoclonal antibodies as candidate 

therapeutics against emerging viruses. Front Med. 2017;11:462-470.

4. National Health Commission of China. Diagnosis and treatment guideline for covid-19 pneumonia (seventh edition). Accessed March,14 2020. http://www.nhc.gov.cn/xcs/zhengcwj/202003/46c9294a7dfe4cef80dc7f5912eb1989.shtml. antibodies: a review. Curr Clin Pharmacol. 2018;13:85-99.

6. Ecker DM, Jones SD, Levine HL. The therapeutic monoclonal antibody market. MABSAUSTIN. 2015;7:9-14.

7. ACTIP. Monoclonal antibodies approved by the ema and fda for therapeutic use. Accessed November, 23 2019. http://www.actip.org/products/monoclonal-antibodies-approved-by-the-emaand-fda-for-therapeutic-use/.

8. Walsh G. Biopharmaceutical benchmarks 2018. NAT BIOTECHNOL. 2018;36:1136-1145. drugs in china: a look from policy perspectives. HUM VACC IMMUNOTHER. 2019;15:26952705.

10. Peihong X, Rongfu G. Trends of chinese pharmaceutical market based on current medication status in hospitals. World clinical drugs. 2017;38:68-72.

11. PharmacyChecker. Com U.S. Humira price. Accessed November, 262019. https://www.pharmacychecker.com/humira/. 12. The State Council of China. "thirteenth five-year plan" for deepening the medical reforms. Accessed September, 01 2019. http://www.gov.cn/zhengce/content/201701/09/content_5158053.htm.

13. Ministry Of Commerce of China. Statistical analysis report on the operation of china's pharmaceutical distribution industry in 2016. Accessed September, 012019. http://images.mofcom.gov.cn/www/201706/20170614162924487.pdf. 14. National Bureau Of Statistics of China: 2019 china household survey main data. Beijing, China, China Statistics Press, 2019. September, 302018. 
1 http://qy1.sfda.gov.ex2.ipv6.nmpa.gov.cn/datasearchenda/face3/base.jsp?tableId=25\&tableName=

2 TABLE25\&title=\%E5\%9B \%BD\%E4\%BA\%A7\%E8\%8D\%AF\%E5\%93\%81\&bcId=1529047137

361213296322795806604.

4 16. Zhang M, Li J, Hu H, Wang Y. Seizing the strategic opportunities of emerging technologies

5 by building up innovation system: monoclonal antibody development in china. HEALTH RES

6 POLICY SY. 2015;13

7 17. World Health Organization And Health Action International. Measuring medicine prices,

8 availability, affordability and price components, 2nd edition. Accessed January, 012016.

9 http://www.haiweb.org/medicineprices/manual/documents.html.

10 18. Mendis S, Fukino K, Cameron A, Laing R, Filipe AJ, Khatib O, Leowski J, Ewen M. The

11 availability and affordability of selected essential medicines for chronic diseases in six low- and

12 middle-income countries. Bull World Health Organ. 2007;85:279-288.

13 19. Yang H, Dib HH, Zhu M, Qi G, Zhang X. Prices, availability and affordability of essential

14 medicines in rural areas of hubei province, china. HEALTH POLICY PLANN. 2010;25:219-229.

15 20. World Health Organization. Atc/ddd index 2019. Accessed April,01 2019.

16 https://www.whocc.no/atc_ddd_index/.

17 21. World Health Organization. Who collaborating centre for drug statistics methodology.

18 Accessed January 012019.

19 https://www.whocc.no/atc_ddd_methodology/who_collaborating_centre/.

22. Jiang X, Wang $\mathrm{Y}, \mathrm{Xu} \mathrm{W}$, Yang S. Comparison of special drug negotiation mechanism under medical insurance in 3 provinces. Chinese Health Economics. 2017;36:40-42.

23. Xi X, Li W, Li J, Zhu X, Fu C, Wei X, Chu S. A survey of the availability, prices and affordability of essential medicines in jiangsu province, china. BMC HEALTH SERV RES. $2015 ; 15: 345$.

24. Liu C, Zhang X, Liu C, Ewen M, Zhang Z, Liu G. Insulin prices, availability and affordability: a cross-sectional survey of pharmacies in hubei province, china. BMC HEALTH SERV RES. 2017;17:597. 


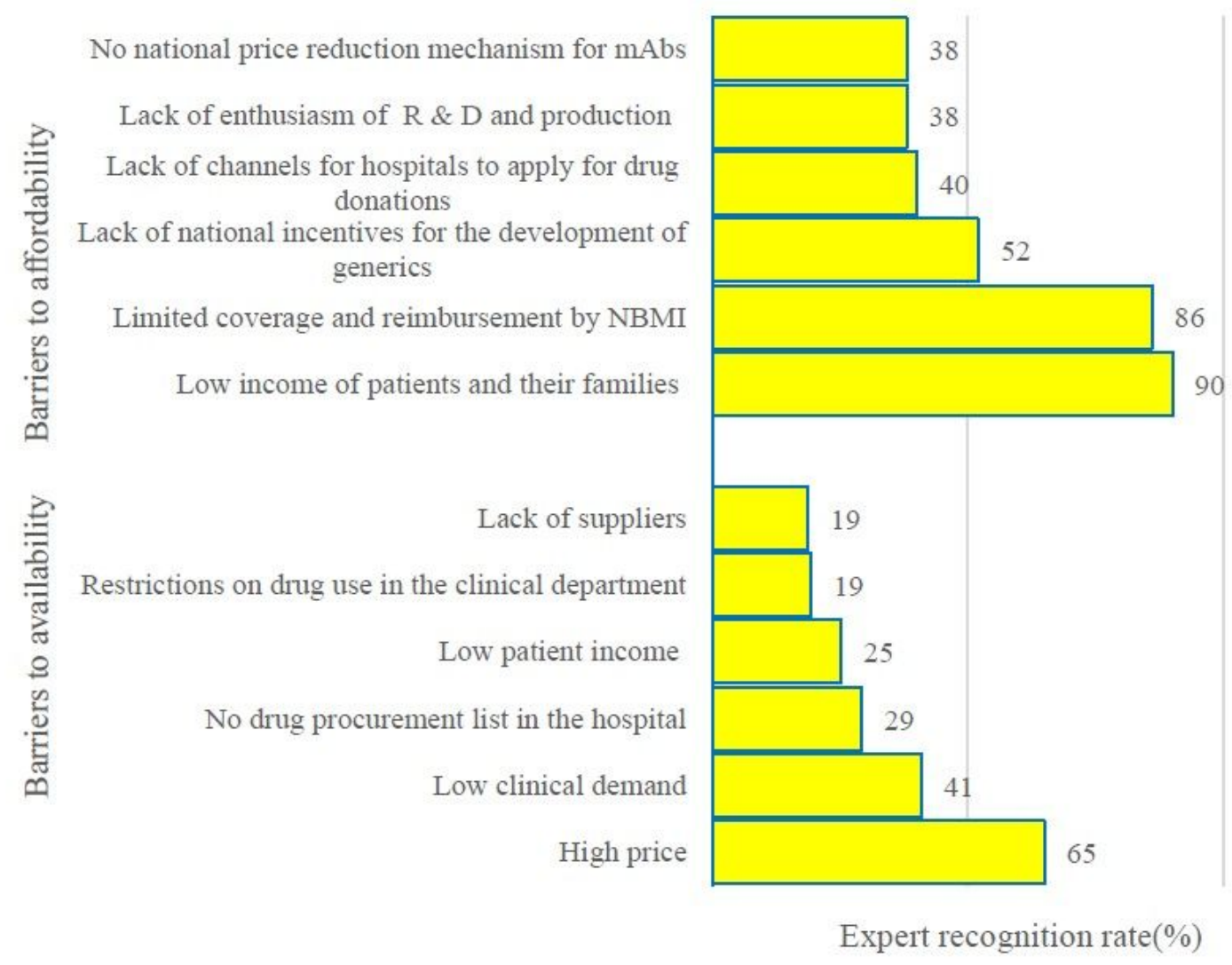

\section{Figure 1}

Main barriers to availability and affordability of 13 therapeutic mAbs 


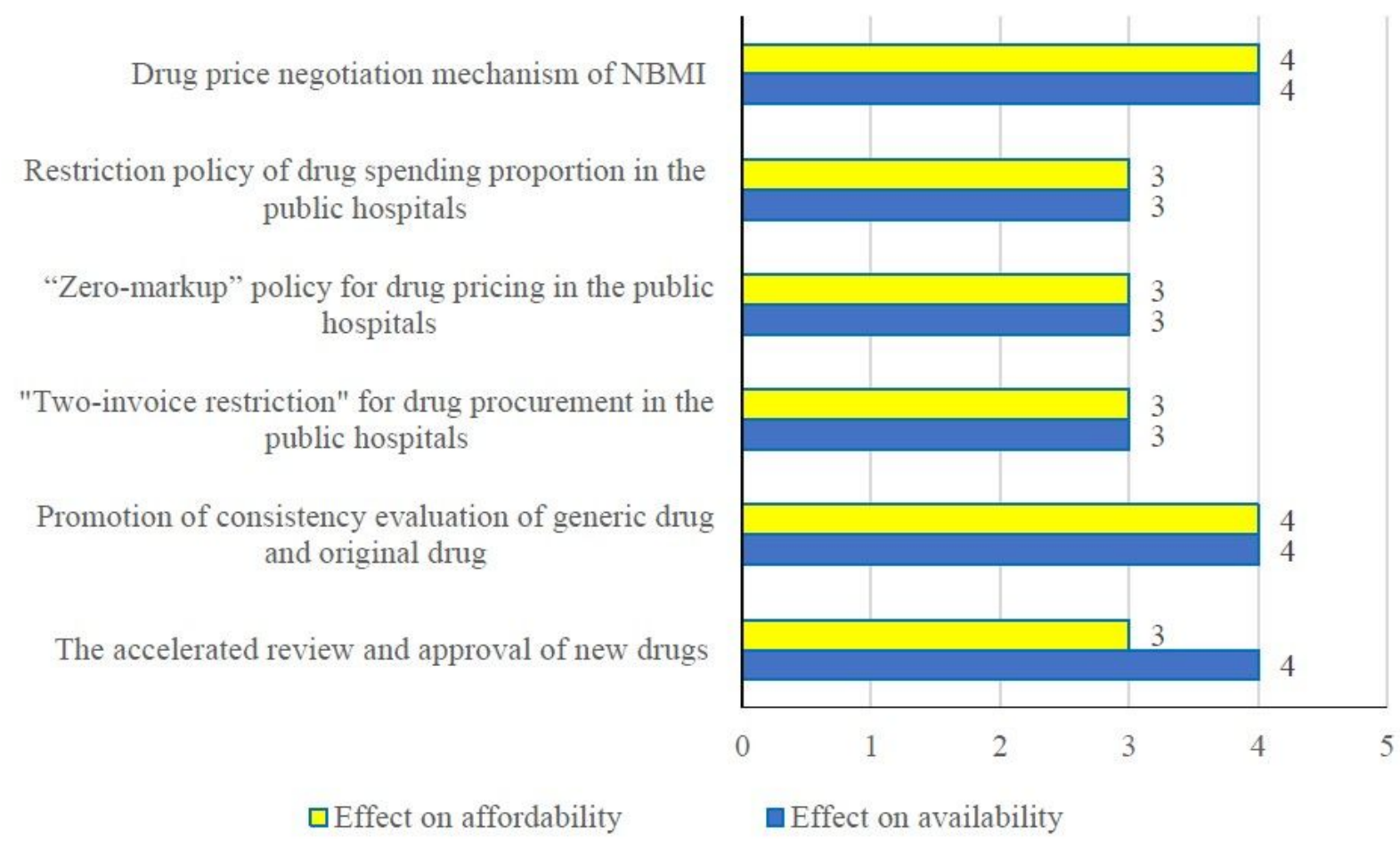

(Completely useless $=1$, Useless $=2$, Somewhat useful $=3$, Useful $=4$, Very useful $=5$ )

\section{Figure 2}

Main barriers to availability and affordability of 13 therapeutic mAbs

\section{Supplementary Files}

This is a list of supplementary files associated with this preprint. Click to download.

- Tables.pdf

- AdditionalFiles.pdf

- Tables.pdf

- AdditionalFiles.pdf 